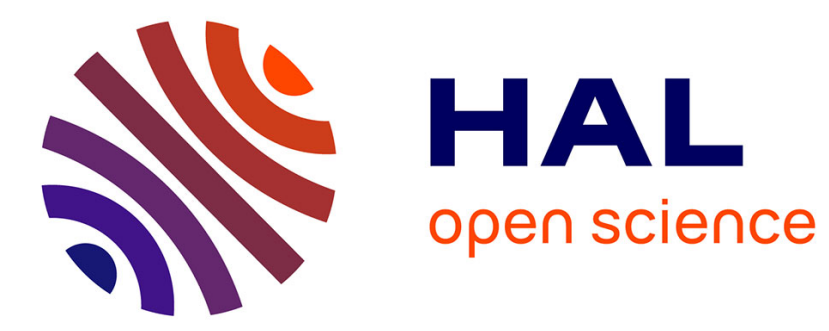

\title{
Global stabilizing feedback law for a problem of biological control of mosquito-borne diseases
}

Pierre-Alexandre Bliman, Maria Soledad Aronna, Flávio C. Coelho, Moacyr da Silva

\section{- To cite this version:}

Pierre-Alexandre Bliman, Maria Soledad Aronna, Flávio C. Coelho, Moacyr da Silva. Global stabilizing feedback law for a problem of biological control of mosquito-borne diseases. 54th IEEE Conference on Decision and Control, Dec 2015, Osaka, Japan. hal-01261162

\section{HAL Id: hal-01261162 \\ https://hal.inria.fr/hal-01261162}

Submitted on 24 Jan 2016

HAL is a multi-disciplinary open access archive for the deposit and dissemination of scientific research documents, whether they are published or not. The documents may come from teaching and research institutions in France or abroad, or from public or private research centers.
L'archive ouverte pluridisciplinaire HAL, est destinée au dépôt et à la diffusion de documents scientifiques de niveau recherche, publiés ou non, émanant des établissements d'enseignement et de recherche français ou étrangers, des laboratoires publics ou privés. 


\title{
Global stabilizing feedback law for a problem of biological control of mosquito-borne diseases
}

\author{
Pierre-Alexandre Bliman ${ }^{1,2}$ and M. Soledad Aronna ${ }^{1}$ and Flávio C. Coelho ${ }^{1}$ and Moacyr A.H.B. da Silva ${ }^{1}$
}

\begin{abstract}
The control of the spread of dengue fever by introduction of the intracellular parasitic bacterium Wolbachia in populations of the vector Aedes aegypti, is presently one of the most promising tools for eliminating dengue, in the absence of an efficient vaccine. The success of this operation requires locally careful planning to determine the adequate number of mosquitoes carrying the Wolbachia parasite that need to be introduced into the natural population. The latter are expected to eventually replace the Wolbachia-free population and guarantee permanent protection against the transmission of dengue to human.

In this paper, we propose and analyze a model describing the fundamental aspects of the competition between mosquitoes carrying Wolbachia and mosquitoes free of the parasite. We then introduce a simple feedback control law to synthesize an introduction protocol, and prove that the population is guaranteed to converge to a stable equilibrium where the totality of mosquitoes carry Wolbachia. The techniques are based on the theory of monotone control systems, as developed after Angeli and Sontag. Due to bistability, the considered inputoutput system has multivalued static characteristics, but the existing results are unable to prove almost-global stabilization, and ad hoc analysis has to be conducted.

Keywords: Wolbachia, Global stabilization, Monotone systems, Input-output characteristic
\end{abstract}

\section{INTRODUCTION}

\section{A. Arboviroses and vector control}

Arboviruses (arthropod borne viruses) are viruses transmitted to humans by arthropod, such as the mosquito. They are pathogens of many and important diseases, putting at risk considerable portions of the human population, and infecting millions of people every year. Mosquitoes (Culicidae family of the insects) are a huge public health concern as they are vectors of many arboviroses such as yellow fever, dengue and chikungunya.

The control of these diseases can be achieved by acting on the population of mosquitoes, and in absence of vaccine or curative treatment, it is essentially the only feasible way. Application of insecticides for both adults and larvae and mechanical removal of breeding sites are the most popular strategies to control the population of mosquitoes. The intensive use of insecticides, however, has negative impacts for humans, animals and the environment. Besides, the gradual increase of mosquito resistance to insecticides

\footnotetext{
${ }^{1}$ All authors are with Escola de Matemática Aplicada, Fundação Getulio Vargas, Praia de Botafogo 190, 22250-900 Rio de Janeiro - RJ, Brazil. E-mails: pierre-alexandre.blimaneinria.fr; aronna@impa.br; fccoelhodfgv.br;

2P.-A. Bliman is also with Inria, Domaine de Voluceau, Rocquencourt BP105, 78153 Le Chesnay cedex, France.
}

usually leads to partial or complete decrease of the efficiency of this strategy in the long run [1], [2]. Alternative or supplementary vector control strategies have been proposed and implemented, such as the release of transgenic or sterile mosquitoes [3], [4]. Notice that an intrinsic weakness of the techniques listed above lies paradoxically in the fact that they aim at the local eradication of the vector, whose disappearance offers no protection against subsequent reinvasions.

Recently, the release of Aedes aegypti mosquitoes infected by the bacterium Wolbachia has been proposed as a promising strategy to control dengue and chikungunya, due to the fact that this bacterium limits severely the vectorial competence of Aedes aegypti. The international program Eliminate Dengue [5] is currently testing in the field this strategy, in several locations around the world: Australia, Indonesia, Vietnam, Colombia and Brazil. The release of infected mosquitoes with Wolbachia has the advantages of being safe for humans and the environment and inexpensive when compared to other control strategies [6].

\section{B. Wolbachia sp. as a biological control tool}

Wolbachia sp. is a genus of bacteria that is a common intracellular parasite of many species of arthropods. It is often found in anthropophilic mosquitoes such as Aedes albopictus or Culex quinquefasciatus but there is no report of Aedes aegypti naturally infected by this bacterium [7].

There is evidence that the spread of certain strains of Wolbachia in populations of Aedes aegypti drastically reduces the vector competence of the mosquito for dengue and other diseases [8], [9], [10]. Some strains of Wolbachia reduce the lifespan of the mosquito, consequently limiting the proportion of surviving mosquitoes at the completion of the incubation period. More importantly, Wolbachia appears to decrease the virulence of the dengue infection in the mosquitoes, increasing the incubation period or blocking the virus, which also reduces the overall vector competence.

The infestation of natural Aedes aegypti populations by Wolbachia-contaminated strains can be achieved by releasing in the field a large number of Wolbachia-infected mosquitoes bred in laboratory. Experiments have been conducted successfully in Australia [11], Vietnam, Indonesia and are currently being applied in Brazil, within the Eliminate Dengue Program. In these experiments, the introduction of a number of Wolbachia-infected mosquitoes in the population triggered a Wolbachia outbreak whose outcome was the fixation of the bacteria in the population, with more than $90 \%$ of prevalence. The effects of this fixation of Wolbachia on the dynamics of dengue in the field is currently under investigation, but 


\begin{tabular}{|c||c|c|}
\hline & Uninfected $\sigma^{\top}$ & Infected $\sigma^{\top}$ \\
\hline \hline Uninfected $q$ & Uninfected & Sterile eggs \\
\hline Infected $q$ & Infected & Infected \\
\hline
\end{tabular}

TABLE I

CYTOPLASMIC INCOMPATIBILITY AND VERTICAL TRANSMISSION OF Wolbachia BACTERIA. THE STATE OF THE OFFSPRING IS INDICATED, DEPENDING ON THE PARENTS STATUS

preliminary results are encouraging [12]. If tractable in practice, this method has certainly the advantage of offering certain robustness to subsequent invasion of Wolbachia-free mosquitoes.

Several mathematical models of the dynamics of invasion of Wolbachia in a population of mosquitoes have been proposed, with distinct objectives. For example, [13] describes a simple model with a single differential equation, sufficient to reveal the bistable nature of the Wolbachia dynamics. Models for spatial dispersion are analyzed in [14] and [15]. In [16], [17], models are presented that assess the effect of the Wolbachia in dengue dynamics. [18] describes a data driven model suitable to estimate accurately some biological parameters by fitting the model with field and lab data. The model presented here is a simplified version of the latter.

\section{Description of the problem and contribution}

A key question about the introduction of Wolbachia in wild mosquitoes concerns the effective strategies of release of infected mosquitoes in the field that can be applied with limited cost to reach the desired state of $100 \%$ of Wolbachiacarrying mosquitoes. In this paper we propose and analyze a simple model of the dynamics of Wolbachia, that allows to investigate these strategies.

The main features of the natural dynamics of Wolbachia that have to be present in the model are the vertical transmission and the peculiar interference on the reproductive outcomes induced by cytoplasmic incompatibility [19]. The transmission of Wolbachia occurs only maternally (i.e. from mother to the offspring), there is no transmission by contact. Cytoplasmic incompatibility (CI) occurs when a female uninfected by Wolbachia is inseminated by an infected male, a mating that leads to sterile eggs. It provides a benefit to infected females against uninfected ones and therefore facilitates the Wolbachia spread. The strains $w \mathrm{Mel}$ and $w$ MelPop of Wolbachia that are being used in the field experiments with Aedes aegypti induce almost total CI [20]. Table I schematizes the results of the mating of infected and uninfected mosquitoes when the $\mathrm{CI}$ is $100 \%$. The model that we propose below captures all of these features and is simple enough to allow for a fairly complete analysis.

The corresponding system is shown to possess two unstable equilibria, which correspond to extinction of the two populations and a coexistence equilibrium; and two locally asymptotically stable equilibria, which correspond to Wolbachia-free and complete infestation equilibria. Using the fact that measurements are achieved and available during the whole release process, we propose a simple feedback control law to compute the released quantity of infected mosquitoes. The key result of the paper (Theorem 7) proves that, in the presence of mosquitoes, this control law has the capacity to asymptotically settle the bacterium from whatever initial conditions, and in particular from the completely Wolbachiafree equilibrium. Of course, a major benefit of feedback compared to open-loop approaches is its ability to cope with the uncertainties on the model dynamics (e.g. in the modeling of the life stages and the population structure), on the parameters (population size, mortality, reproductive rates, etc.), and on the conditions of the realization (in particular on the size of the population to be treated).

Up to our knowledge, this constitutes the first attempt to use feedback approach for introduction of Wolbachia within a population of arthropods. We only treat here the case of the release of Wolbachia-positive larvae and full information on the quantity of Wolbachia-negative larvae. Yet, the same dynamical model offers the ability to study other configurations, both for control and observation, and the corresponding issues will be examined in future work.

The paper is organized as follows. The model is introduced in Section II, and normalized. Analysis of the uncontrolled model is made in Section III, showing the announced bistability between Wolbachia-free equilibrium and full infestation. A proportional control law is then proposed in Section IV, that leads to global stability of the full infestation equilibrium. Simulations are shown in Section $\mathrm{V}$ and concluding remarks achieve the text in Section VI. The reader is referred to [21] for details and complete proofs.

Notation. For $n \in \mathbb{N}, \mathbb{R}^{n}$ denotes the $n$-dimensional Euclidean real space, and $\mathbb{R}_{+}^{n}$ the cone of vectors in $\mathbb{R}^{n}$ with nonnegative components. We write $\max \{a ; b\}$ (resp. $\min \{a ; b\}$ ) for maximum (resp. minimum) of two real numbers $a, b$. Also, for a closed convex cone $\mathcal{K} \subset \mathbb{R}^{n}$ with nonempty interior int $\mathcal{K}, x \gg_{\mathcal{K}} x^{\prime}$ means $x-x^{\prime} \in$ int $\mathcal{K}$. Last, we write LAS (resp. GAS) for locally (resp. globally) asymptotically stable.

\section{A SIMPLE MODEL OF INFESTATION BY Wolbachia}

The simplified compartment model we introduce includes two life stages: a preliminary one, gathering the aquatic phases (egg and larva) where the mosquitoes are subject to space and food competition; and an adult one, representing all the posterior aerial phases (pupae, immature and mature adult). Accordingly, we will denote $\mathbf{L}$ and $\mathbf{A}$ the corresponding state variables. The uninfected and infected (by Wolbachia) populations will be distinguished by indexes $U$ and $W$ respectively, so we end up with a four state variables model, namely $\mathbf{L}_{U}, \mathbf{L}_{W}$ and $\mathbf{A}_{U}, \mathbf{A}_{W}$, that represent the numbers of uninfected, resp. infected, vectors in preliminary and adult phases. The parameters are positive and have the following meaning, $\alpha_{U}, \alpha_{W}$ : fecundity rates of uninfected and infected insects; $\nu$ : rate of transfer from the preliminary to the adult stage; $\mu$ : mortality rate of uninfected and infected insects in preliminary stage; $\mu k$ : characteristic of the 
additional competition-induced mortality rate in preliminary stage; $\mu_{U}, \mu_{W}$ : mortality rates of uninfected and infected insects at adult stage. The model is as follows.

$$
\begin{gathered}
\dot{\mathbf{L}}_{U}=\alpha_{U} \frac{\mathbf{A}_{U}}{\mathbf{A}_{U}+\mathbf{A}_{W}} \mathbf{A}_{U}-\nu \mathbf{L}_{U}-\mu\left(1+k\left(\mathbf{L}_{W}+\mathbf{L}_{U}\right)\right) \mathbf{L}_{U} \\
\dot{\mathbf{A}}_{U}=\nu \mathbf{L}_{U}-\mu_{U} \mathbf{A}_{U} \\
\dot{\mathbf{L}}_{W}=\alpha_{W} \mathbf{A}_{W}-\nu \mathbf{L}_{W}-\mu\left(1+k\left(\mathbf{L}_{W}+\mathbf{L}_{U}\right)\right) \mathbf{L}_{W}+\mathbf{u}
\end{gathered}
$$

$$
\dot{\mathbf{A}}_{W}=\nu \mathbf{L}_{W}-\mu_{W} \mathbf{A}_{W}
$$

The difference between the two populations lies in the different fecundity and mortality rates. The mortality during the larva stage and the duration of the latter are considered unmodified by the disease. Also, the (quadratic) competition term is assumed to act equally on both populations, with an effect proportional to the global number of larvae.

The first effect of Wolbachia is to diminish fertility and life duration, leading to reduced fitness for the infected mosquitoes. This assumption will correspond to the choice of parameters made in (4) below. The second effect, namely the cytoplasmic incompatibility, modeled here as complete, forbids fecund mating between infected males and uninfected females. This is rendered in (1a) by a birth term proportional altogether to the number and to the ratio of uninfected adults. Last, notice the input variable $\mathbf{u}$ in equation (1c), that models the on-purpose introduction of infected larvae in the system in order to settle Wolbachia.

The previous model is now normalized, see [21] for details. The normalized model, used in the sequel, is:

$$
\begin{gathered}
\dot{L}_{U}=\gamma_{U} \mathcal{R}_{0}^{U} \frac{A_{U}}{A_{U}+A_{W}} A_{U}-\left(1+L_{W}+L_{U}\right) L_{U} \\
\dot{A}_{U}=L_{U}-\gamma_{U} A_{U} \\
\dot{L}_{W}=\gamma_{W} \mathcal{R}_{0}^{W} A_{W}-\left(1+L_{W}+L_{U}\right) L_{W}+u \\
\dot{A}_{W}=L_{W}-\gamma_{W} A_{W}
\end{gathered}
$$

with the choice of dimensionless parameters

$$
\gamma_{\eta}:=\frac{\mu_{\eta}}{\nu+\mu}, \quad \mathcal{R}_{0}^{\eta}:=\frac{\nu \alpha_{\eta}}{(\nu+\mu) \mu_{\eta}}, \quad \eta=U, W .
$$

The state variable for system (2) is denoted $x:=$ $\left(L_{U}, A_{U}, L_{W}, A_{W}\right)$, and for sake of simplicity (2) is written $\dot{x}=f(x)+B u$, with $B:=\left(\begin{array}{llll}0 & 0 & 1 & 0\end{array}\right)^{\top}$ and

$$
f(x):=\left(\begin{array}{c}
\gamma_{U} \mathcal{R}_{0}^{U} \frac{A_{U}}{A_{U}+A_{W}} A_{U}-\left(1+L_{W}+L_{U}\right) L_{U} \\
L_{U}-\gamma_{U} A_{U} \\
\gamma_{W} \mathcal{R}_{0}^{W} A_{W}-\left(1+L_{W}+L_{U}\right) L_{W} \\
L_{W}-\gamma_{W} A_{W}
\end{array}\right) .
$$

We assume in the remainder of the paper

$$
\mathcal{R}_{0}^{U}>\mathcal{R}_{0}^{W}>1
$$

Ensuring sustainability of each of the two isolated populations, with even better sustainability for the non-infected one, (4) is coherent with the experimental observations.

\section{ANALYSIS OF THE UNCONTROLLED SYSTEM}

\section{A. Well-posedness, positivity and boundedness}

One first shows the well-posedness of the Cauchy problem associated to the uncontrolled (zero-input) system

$$
\dot{x}=f(x)
$$

Theorem 1: For any initial value in $\mathbb{R}_{+}^{4}$, there exists a unique solution to the initial value problem associated to system (5). The latter is defined on $[0,+\infty)$, depends continuously on the initial conditions and takes on values in $\mathbb{R}_{+}^{4}$. Moreover, it is uniformly ultimately bounded.

\section{B. Monotonicity}

Noticing that the presence of the infected population cripples the growth of the uninfected one and vice versa, yields the following fundamental result [21].

Theorem 2: System (5) is strongly order-preserving in $\mathbb{R}_{+}^{4}$ for the order induced by the cone $\mathcal{K}:=\mathbb{R}_{-} \times \mathbb{R}_{-} \times \mathbb{R}_{+} \times \mathbb{R}_{+}$, defined by: $x \geq \mathcal{K} x^{\prime} \Leftrightarrow x_{i} \leq x_{i}^{\prime}, i=1,2$ and $x_{i} \geq x_{i}^{\prime}, i=$ 3,4 .

Definitions of monotone and strongly order-preserving semiflows defined on a topological space $X$ partially ordered by an order relation $\leq_{\mathcal{K}}$ generated by a cone $\mathcal{K}$, can be found in [22]. System (5) is therefore monotone in $\mathbb{R}_{+}^{4}$, but not strongly monotone: indeed, the trajectories departing from the sets $\left\{x \in \mathbb{R}_{+}^{4}: L_{U}=0, A_{U}=0\right\}$ and $\left\{x \in \mathbb{R}_{+}^{4}: L_{W}=0, A_{W}=0\right\}$ remain in these sets, and consequently do not verify the strict ordering property for the two null components.

\section{Equilibrium points and stability}

The next result [21] describes the situation of the equilibrium points and their stability.

Theorem 3: System (5) possesses four equilibrium points, denoted $x_{0,0}, x_{U, 0}, x_{0, W}$ and $x_{U, W}$ and corresponding respectively to zero population, disease-free state, complete infestation, and coexistence. Moreover, the latter fulfill the following inequalities: $x_{U, 0} \ll \mathcal{K} \quad x_{U, W} \quad \ll \mathcal{K} \quad x_{0, W}$, and $x_{U, 0} \ll_{\mathcal{K}} x_{0,0} \ll \mathcal{K} x_{0, W}$. Last, the equilibria $x_{U, 0}$ and $x_{0, W}$ are LAS, while the two other ones are unstable.

\section{Positively invariant sets and basins of attraction}

The order relations between the equilibria (see Theorem 3 ) yield supplementary information on some invariant sets.

Theorem 4: The order interval $\llbracket x_{U, 0} ; x_{0, W} \rrbracket_{\mathcal{K}}:=$ $\left\{x \in \mathbb{R}^{4}: x_{U, 0} \leq_{\mathcal{K}} x \leq_{\mathcal{K}} x_{0, W}\right\} \subset \mathbb{R}_{+}^{4}$ is positively invariant for system (5). Moreover, the order interval $\llbracket x_{U, W} ; x_{0, W} \rrbracket \mathcal{K}$ (resp. $\llbracket x_{U, 0} ; x_{U, W} \rrbracket \mathcal{K}$ ) is contained in the basin of attraction of $x_{0, W}$ (resp. $x_{0, U}$ ).

\section{ANALYSIS OF THE CONTROLLED SYSTEM}

\section{A. A class of static output-feedback control laws}

The feedback law $u=K L_{U}$ is considered in the sequel, for adequate (positive) values of the scalar gain $K$. Writing $e:=\left(\begin{array}{llll}1 & 0 & 0 & 0\end{array}\right)^{\top}$, one obtains the closed-loop system:

$$
\dot{x}=f(x)+K B e^{\top} x,
$$


or in developed form:

$$
\begin{gathered}
\dot{L}_{U}=\gamma_{U} \mathcal{R}_{0}^{U} \frac{A_{U}}{A_{U}+A_{W}} A_{U}-\left(1+L_{W}+L_{U}\right) L_{U} \\
\dot{A}_{U}=L_{U}-\gamma_{U} A_{U} \\
\dot{L}_{W}=\gamma_{W} \mathcal{R}_{0}^{W} A_{W}-\left(1+L_{W}+L_{U}\right) L_{W}+K L_{U} \\
\dot{A}_{W}=L_{W}-\gamma_{W} A_{W}
\end{gathered}
$$

Theorem 5: For any initial value in $\mathbb{R}_{+}^{4}$, there exists a unique solution to the initial value problem associated to system (6). The latter is defined on $[0,+\infty)$, depends continuously on the initial conditions and takes on values in $\mathbb{R}_{+}^{4}$. Moreover, it is uniformly ultimately bounded.

\section{B. Equilibrium points and critical gain}

The next result shows that, for gains larger than certain critical value, the only LAS equilibrium is $x_{0, W}$. Moreover, the value of this critical gain depends only upon the basic offspring numbers of the two populations and the ratio between their mortality rates, which are all scale-free information.

Theorem 6: If $K>K^{*}:=\frac{\gamma_{W}}{\gamma_{U}}\left(\sqrt{\mathcal{R}_{0}^{U}}-\sqrt{\mathcal{R}_{0}^{W}}\right)^{2}$, then the closed-loop system (6) possesses two equilibria, namely $x_{0,0}$ and $x_{0, W}$, and their local stability properties are not modified: $x_{0,0}$ is unstable and $x_{0, W}$ is LAS.

\section{Global stability issues}

We now turn to the most innovative part of this paper, namely the global behavior of the closed-loop system (6). The result we state here shows that the introduction of infected larvae according to the proposed proportional feedback law yields conclusive infestation when the gain is larger than the critical value. More precisely, we have the following convergence result.

Theorem 7: If $K>K^{*}$, all trajectories of system (6) issuing from a point in $\mathbb{R}_{+}^{4}$ distinct from $x_{0,0}$ converge towards the complete infestation equilibrium $x_{0, W}$.

Strictly speaking, Theorem 7 is an almost global convergence result: complete infestation occurs, except for a zero measure set of initial conditions. However, in the present case, this set is reduced to the unstable equilibrium.

Two attempts to prove Theorem 7 are rapidly presented in Sections IV-C.1 and IV-C.2. Their interest is to show how quite natural approaches fail to provide information on the asymptotic behavior: a new approach is therefore needed. A conclusive method is exposed in Section IV-C.3.

1) Global stability of a singularly perturbed system, by LaSalle's invariance principle: We present a first attempt, based on Lyapunov techniques. Consider the simpler system

$$
\begin{gathered}
\dot{L}_{U}=\mathcal{R}_{0}^{U} \frac{\gamma_{W} L_{U}}{\gamma_{W} L_{U}+\gamma_{U} L_{W}} L_{U}-\left(1+L_{W}+L_{U}\right) L_{U} \\
\dot{L}_{W}=\mathcal{R}_{0}^{W} L_{W}-\left(1+L_{W}+L_{U}\right) L_{W}+K L_{U}
\end{gathered}
$$

System (8) is deduced from (7) by applying singular perturbation, formally putting $0=L_{U}-\gamma_{U} A_{U}, 0=L_{W}-$ $\gamma_{W} A_{W}$. In other words, we assume that (7b) and (7d) are fast dynamics, and (7a) and (7c) are comparatively slower.
Well-posedness and positiveness of system (8) present no difficulties, one states directly its asymptotic properties.

Theorem 8: Assume $K>K^{*}$. Then system (8) possesses two equilibria, which are the projections of $x_{0,0}$ and $x_{0, W}$. The former one is unstable, while the latter one is LAS. Last, all trajectories of system (8) converge towards the LAS one, except the unstable equilibrium itself.

An interesting point is that stability may be obtained by showing that the derivative of the function $V\left(L_{U}, L_{W}\right):=$ $\frac{L_{U}}{L_{U}+L_{W}}$ is nonpositive along the trajectories of (8) when $K=K^{*}$ [21]. While this Lyapunov function is quite appealing, it has not proved possible to extend this idea to the complete controlled system (6).

2) A monotone control system perspective: The second method now explored is an attempt to apply the results on monotone control systems, as worked out in particular by [23], see also [24], [25], [26], [27]. The principle of this approach consists in decomposing the system under study as a monotone input-output system with feedback. Given the fact (see Theorem 2) that the uncontrolled system is monotone, a most natural way to do this is to write system (6) as

$$
\begin{gathered}
\dot{x}=f(x)+K B u, \quad y=L_{U}=e^{\top} x \\
u=y
\end{gathered}
$$

Arguing as in Theorem 2, one shows that the input-to-state map $u \mapsto x$ given by (9a) is monotone when the state space is endowed with the ordering $\leq_{\mathcal{K}}$; while the state-to-output map $x \mapsto y=e^{\top} x=L_{U}$ is anti-monotone. We are thus in a configuration of monotone system with negative feedback.

In such a case, the study of asymptotics of the system obtained when closing the loop by the unitary feedback (9b) can be done by introducing static characteristics [23], [27]. However, things become immediately complicated in

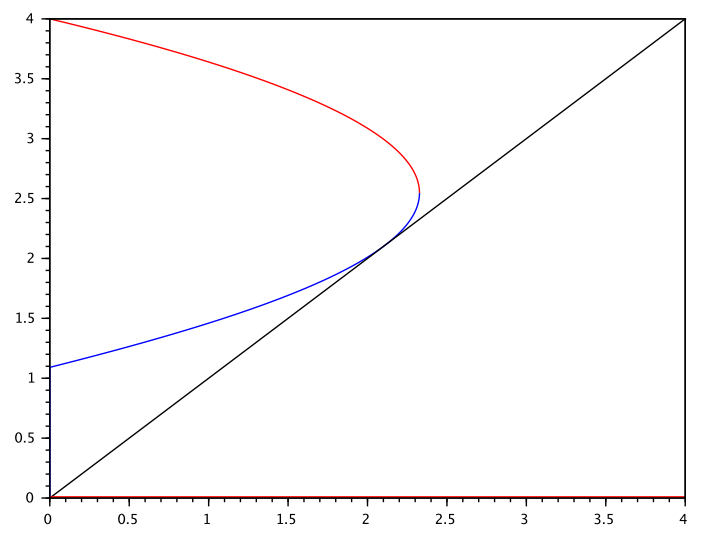

Fig. 1. The multivalued input-output characteristic $\bar{u} \mapsto k(\bar{u})$ corresponding to input-output system (9a) (in red) and the diagonal $\bar{u} \mapsto \bar{u}$ (in black), in the critical case $K^{*}=K$. See text for explanations

the case of system (9): constant input $\bar{u}=0$ leads to the uncontrolled system (5), which has been proved to possess 
two LAS equilibria (and two unstable ones). In such a case, one can consider multivalued input-state and input-output characteristics, as made in [28], [29]. The corresponding input-output characteristic is drawn in red in Figure 1 (for the parameter values given in Section $\mathrm{V}$ below) and for the corresponding critical value $K^{*}$ of $K$.

As seen in the figure, the input-output characteristic has basically two branches (both drawn in red). The first one merges with the horizontal axis: it corresponds to a branch of equilibria with null value of the output $y=L_{U}$ that departs from $x_{0, W}$ for $\bar{u}=0$. The second one is a decreasing curve, defined for values of $\bar{u}$ ranging from zero to a value close to 2.32: it corresponds to the output value of a branch of equilibria departing from $x_{U, 0}$. The blue curve, which does not pertain to the input-output characteristic, indicates the output values of a branch of unstable equilibria originating from $x_{U, W}$, that vanishes together with the upper curve. The diagonal line (that determines the fixed points of $k$ ) is shown, tangent to the blue curve due to the fact that $K=K^{*}$ here.

For $K>K^{*}$, the complete infestation equilibrium is therefore the only fixed point of the multivalued map $k$. But the iterative sequences $\bar{u}_{k+1}=k\left(\bar{u}_{k}\right)$ do not converge systematically towards this point, and the only information that can be deduced in case of multivalued inputoutput characteristic [28], [29] is that all trajectories are bounded and that the output $y=L_{U}$ fulfills the inequalities: $0 \leq \limsup _{t \rightarrow+\infty} y(t) \leq k^{\sup }\left(\liminf _{t \rightarrow+\infty} y(t)\right)$, where $k^{\sup }(\bar{u}):=$ $\max _{y \in k(\bar{u})} y$. As a conclusion, the decomposition (9), that seemed

a natural framework to analyze the behavior of the controlled system (6) immediately fails to produce a global vision of the asymptotic behavior.

3) Monotonicity revisited and proof of the global stability: A crucial step in the proof of Theorem 7 is the introduction of a decomposition of system (6) different from (9). Let $|z|_{ \pm}:=z$ if $\pm z \geq 0,0$ otherwise. Clearly, $z=$ $|z|_{-}+|z|_{+}, z \in \mathbb{R}$. Consider now the input-output system

$$
\begin{gathered}
\dot{L}_{U}=\gamma_{U} \mathcal{R}_{0}^{U} \frac{A_{U}}{A_{U}+A_{W}} A_{U}-\left(1+L_{W}+L_{U}\right) L_{U} \\
\dot{A}_{U}=L_{U}-\gamma_{U} A_{U} \\
\dot{L}_{W}=\gamma_{W} \mathcal{R}_{0}^{W} A_{W}-(10 \mathrm{a}) \\
\dot{A}_{W}=L_{W}-L_{W}+\mid K-L_{W} A_{W} L_{U}+K u \\
y=\left|1-\frac{L_{W}}{K}\right|_{+} L_{U}
\end{gathered}
$$

One verifies easily that closing (10) by $u=y$ yields (6). The following result is a key step, see [21] for further details.

Lemma 9: The input-output system (10) is monotone with negative feedback, when the state space is endowed with the order $\geq_{\mathcal{K}}$ defined in Theorem 2 .

\section{NUMERICAL SIMULATIONS}

Illustrative simulations are presented, with the following realistic parameters: $\gamma_{U}=0.8, \gamma_{W}=1, \mathcal{R}_{0}^{U}=5, \mathcal{R}_{0}^{W}=3$. Notice that the mortality is higher for the Wolbachia infected population $\left(\gamma_{U}<\gamma_{W}\right)$, and its sustainability inferior $\left(\mathcal{R}_{0}^{U}>\right.$ $\mathcal{R}_{0}^{W}$ ). Computation of the critical gain yields $K^{*} \simeq 0.318$.

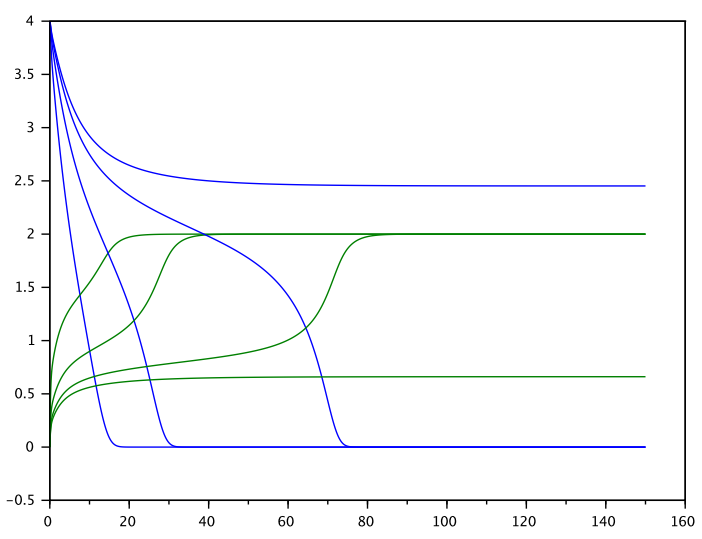

Fig. 2. Evolution of $L_{U}(t)$ and $L_{W}(t)$ as functions of time, for $K=1$, $K=0.5, K=0.35$ and $K=0.3$. See the text for details

Figures 2 shows on the same diagram the evolution of the state variables $L_{U}$ (in blue) and $L_{W}$ (in green) as functions of time, for different values of the gain. The initial state is the Wolbachia-free equilibrium $x_{U, 0}$, and $K$ is respectively chosen to be $1,0.5,0.35$ and 0.3 . For the three first values, settling of complete infestation is slower when the gain decreases. For the last value, slightly smaller than the critical value $K^{*} \simeq 0.318$, a coexistence equilibrium appears asymptotically, indicating that the bound $K^{*}$ is tight.

\section{CONCLUSIONS AND FURTHER STUDIES}

We presented and analyzed a model for the infestation by bacterium Wolbachia of a population of mosquitoes typically one of the genera Aedes involved in the transmission of arboviroses such as yellow fever, dengue fever or chikungunya. A method of implementation based on the introduction of a quantity of contaminated insects proportional to the size of the healthy population was proposed and shown, analytically and by simulation, to be capable of successfully spreading the bacteria, provided the gain is sufficiently large. This feedback method requires continuous measurement of the population. Its main interest with respect to the release(s) of a predefined quantity, is the reduction of the number of released mosquitoes, and thus of the treatment cost, without jeopardizing the success of the introduction of the bacteria - something which can happen e.g. in case of underestimation of the initial population size. To our knowledge, this is the first use of the control theory notion of feedback in such a context.

Among other steps leading to application, the adaptation to effective conditions has to be done. First, the model presented here has been chosen continuous in time for simplicity, but passing to discrete-time system seems to present a priori no difficulties. Also, the present framework assumes the measurement of a larva stage of the healthy portion of the 
population, and as well the release of a larva stage of the contaminated one. The practical conditions can be different, and the method can be adapted in consequence (leading though to similar, but different, convergence questions). Last, robustness with respect to parameter uncertainties (or to seasonal variations) has not been tackled here.

An advantage of the present modeling framework is to open the way to comparisons with optimal policies - for example the one that minimizes the total number of released mosquitoes, while succeeding in spreading Wolbachia. This point will be studied in a next future. Also, this framework provides a first basis to consider questions related to strategy improvement by mitigating several control principles, or to the complex phenomena of interaction between different vector species and different arboviruses, that may occur in the context of control of different diseases.

From a mathematical point of view, one of the difficulties of the study is that the system presents two stable equilibria, corresponding to Wolbachia-free situation and complete infestation. While the key arguments are based on the theory of input-output monotone systems developed after [23], none of the posterior refinements to multivalued characteristics or quasi-characteristics allowed to establish formally the main convergence result, and adequate adaptation had to be achieved. Extensions in this direction are presently studied.

\section{ACKNOWLEDGMENT}

The first author is indebted to T. Gedeon for valuable discussions. He was supported by FAPERJ under the APQ5 program. This work was done while the second author was a postdoctoral fellow at IMPA, funded by CAPES-Brazil.

\section{REFERENCES}

[1] R. M. de Freitas and D. Valle, "Challenges encountered using standard vector control measures for dengue in Boa Vista, Brazil," Bulletin of the World Health Organization, vol. 92, no. 9, pp. 685-689, jul 2014.

[2] I. R. Montella, A. J. Martins, P. F. Viana-Medeiros, J. B. P. Lima, I. A. Braga, and D. Valle, "Insecticide resistance mechanisms of Brazilian Aedes aegypti populations from 2001 to 2004," The American journal of tropical medicine and hygiene, vol. 77, no. 3, pp. 467-477, 2007.

[3] L. Alphey, M. Benedict, R. Bellini, G. G. Clark, D. A. Dame, M. W. Service, and S. L. Dobson, "Sterile-Insect Methods for Control of Mosquito-Borne Diseases: An Analysis," Vector-Borne and Zoonotic Diseases, vol. 10, no. 3, pp. 295-311, apr 2010.

[4] L. Alphey, "Genetic Control of Mosquitoes," Annu. Rev. Entomol., vol. 59, no. 1, pp. 205-224, jan 2014.

[5] A. Hoffmann, L. Moreira, S. O'Neill, J. E. Osorio, S. Ritchie, C. Simmons, and M. Turelli. () Eliminate dengue. http://www.eliminatedengue.com/.

[6] J. Popovici, L. A. Moreira, A. Poinsignon, I. Iturbe-Ormaetxe, D. McNaughton, and S. L. O'Neill, "Assessing key safety concerns of a Wolbachia-based strategy to control dengue transmission by Aedes mosquitoes," Memórias do Instituto Oswaldo Cruz, vol. 105, no. 8, pp. 957-964, 2010.

[7] J. L. Rasgon and T. W. Scott, "An initial survey for Wolbachia (Rickettsiales: Rickettsiaceae) infections in selected California mosquitoes (Diptera: Culicidae)," Journal of medical entomology, vol. 41, no. 2, pp. 255-257, 2004.

[8] L. A. Moreira, I. Iturbe-Ormaetxe, J. A. Jeffery, G. Lu, A. T. Pyke, L. M. Hedges, B. C. Rocha, S. Hall-Mendelin, A. Day, M. Riegler, L. E. Hugo, K. N. Johnson, B. H. Kay, E. A. McGraw, A. F. van den Hurk, P. A. Ryan, and S. L. O’Neill, "A Wolbachia Symbiont in Aedes aegypti Limits Infection with Dengue, Chikungunya, and Plasmodium," Cell, vol. 139, no. 7, pp. 1268-1278, dec 2009.
[9] M. S. Blagrove, C. Arias-Goeta, A.-B. Failloux, and S. P. Sinkins, "Wolbachia strain $w$ Mel induces cytoplasmic incompatibility and blocks dengue transmission in Aedes albopictus," Proceedings of the National Academy of Sciences, vol. 109, no. 1, pp. 255-260, 2012.

[10] M. S. Blagrove, C. Arias-Goeta, C. Di Genua, A.-B. Failloux, and S. P. Sinkins, "A Wolbachia $w$ Mel transinfection in Aedes albopictus is not detrimental to host fitness and inhibits Chikungunya virus," PLoS neglected tropical diseases, vol. 7, no. 3, p. e2152, 2013.

[11] A. A. Hoffmann, B. L. Montgomery, J. Popovici, I. Iturbe-Ormaetxe, P. H. Johnson, F. Muzzi, M. Greenfield, M. Durkan, Y. S. Leong, Y. Dong, H. Cook, J. Axford, A. G. Callahan, N. Kenny, C. Omodei, E. A. McGraw, P. A. Ryan, S. A. Ritchie, M. Turelli, and S. L. O'Neill, "Successful establishment of Wolbachia in Aedes populations to suppress dengue transmission," Nature, vol. 476, no. 7361, pp. 454457, aug 2011.

[12] F. D. Frentiu, T. Zakir, T. Walker, J. Popovici, A. T. Pyke, A. van den Hurk, E. A. McGraw, and S. L. O'Neill, "Limited Dengue Virus Replication in Field-Collected Aedes aegypti Mosquitoes Infected with Wolbachia," PLoS Neglected Tropical Diseases, vol. 8, no. 2, p. e2688, feb 2014

[13] M. Turelli, "Cytoplasmic incompatibility in populations with overlapping generations," Evolution, vol. 64, no. 1, pp. 232-241, 2010.

[14] N. Barton and M. Turelli, "Spatial waves of advance with bistable dynamics: cytoplasmic and genetic analogues of Allee effects," The American Naturalist, vol. 178, no. 3, pp. E48-E75, 2011.

[15] P. A. Hancock and H. C. J. Godfray, "Modelling the spread of Wolbachia in spatially heterogeneous environments," Journal of The Royal Society Interface, vol. 9, no. 76, pp. 3045-3054, 2012.

[16] H. Hughes and N. F. Britton, "Modelling the use of Wolbachia to control dengue fever transmission," Bulletin of mathematical biology, vol. 75, no. 5, pp. 796-818, 2013.

[17] M. Z. Ndii, R. Hickson, D. Allingham, and G. Mercer, "Modelling the transmission dynamics of dengue in the presence of Wolbachia," Mathematical biosciences, vol. 262, pp. 157-166, 2015.

[18] J. Koiller, M. Da Silva, M. Souza, C. Codeço, A. Iggidr, and G. Sallet, "Aedes, Wolbachia and Dengue," Inria, France, Research Report RR8462, Jan. 2014.

[19] S. L. O'Neill, A. A. Hoffman, and J. H. Werren, Eds., Influential Passengers: Inherited Microorganisms and Arthropod Reproduction. Oxford University Press, 1998.

[20] T. Walker, P. Johnson, L. Moreira, I. Iturbe-Ormaetxe, F. Frentiu, C. McMeniman, Y. S. Leong, Y. Dong, J. Axford, P. Kriesner et al., "The $w$ Mel Wolbachia strain blocks dengue and invades caged Aedes aegypti populations," Nature, vol. 476, no. 7361, pp. 450-453, 2011.

[21] P.-A. Bliman, M. S. Aronna, F. C. Coelho, and M. A. da Silva, "Ensuring successful introduction of Wolbachia in natural populations of Aedes aegypti by means of feedback control," http://arxiv.org/abs/1503.05216, Tech. Rep., March 2015.

[22] H. L. Smith, Monotone dynamical systems: an introduction to the theory of competitive and cooperative systems, ser. Mathematical surveys and monographs. American Mathematical Society, 1995, vol. 41.

[23] D. Angeli and E. D. Sontag, "Monotone control systems," IEEE Transactions on Automatic Control, vol. 48, pp. 1684-1698, 2003.

[24] J.-L. Gouzé, "A criterion of global convergence to equilibrium for differential systems with an application to Lotka-Volterra systems," Inria, France, Research report 0894, 1988.

[25] C. Cosner, "Comparison principles for systems that embed in cooperative systems, with applications to diffusive Lotka-Volterra models," Dynamics of Continuous, Discrete and Impulsive Systems, vol. 3, pp. 283-303, 1997.

[26] G. Enciso and E. D. Sontag, "Nonmonotone systems decomposable into monotone systems with negative feedback," Journal of Differential Equations, vol. 224, pp. 205-227, 2006.

[27] G. Enciso, "Fixed points and convergence in monotone systems under positive or negative feedback," International Journal of Control, vol. 87, no. 2, pp. 301-311, 2014.

[28] M. Malisoff and P. D. Leenheer, "A small-gain theorem for monotone systems with multi-valued input-state characteristics," IEEE Transactions on Automatic Control, vol. 41, no. 2, pp. 287-292, 2006.

[29] T. Gedeon and G. Hines, "Multi-valued characteristics and Morse decompositions," Journal of Differential Equations, vol. 247, no. 4, pp. 1013-1042, 2009. 